\title{
Educação em saúde durante a pandemia da COVID-19: o que consta no Portal da CAPES
}

\author{
Health education during the COVID-19 pandemic: what appears on the CAPES Portal \\ Educación en salud durante la pandemia de COVID-19: qué hay en el Portal de la CAPES
}

Recebido: 08/09/2021 | Revisado: 15/09/2021 | Aceito: 21/09/2021 | Publicado: 23/09/2021

Vanusa Nascimento Sabino Neves

ORCID: https://orcid.org/0000-0001-6163-1699

Universidade Federal da Paraíba, Brasil

E-mail: pbvanusa@gmail.com

Kellyane Morais Alves

ORCID: https://orcid.org/0000-0001-6730-5333 Hospital Universitário Lauro Wanderley, Brasil

E-mail: kellalves@yahoo.com.br

Lícia Helena Farias Pinheiro

ORCID: https://orcid.org/0000-0002-4555-8409 Hospital Universitário Lauro Wanderley, Brasil E-mail: liciafarias@gmail.com

Silvana Mére Cesário Nóbrega

ORCID: https://orcid.org/0000-0002-0813-1502 Hospital Universitário Lauro Wanderley, Brasil

E-mail: silvanamere@hotmail.com

Vanessa Polyana de Sousa Brito

ORCID: https://orcid.org/0000-0003-1311-7614 Hospital Universitário Lauro Wanderley, Brasil E-mail: polyenfa@hotmail.com

Paula Silva Aragão

ORCID: https://orcid.org/0000-0001-8074-8702 Hospital Universitário Lauro Wanderley, Brasil E-mail: paulasilvaragao@ hotmail.com

\begin{abstract}
Resumo
A educação em saúde é um processo educativo cujo escopo é aumentar a autonomia da população na prevenção do adoecimento, entretanto, a crise atual imposta pela pandemia da COVID-19 tem impactado diversos segmentos sociais. Nessa conjuntura, a disseminação de informações científicas a respeito dessa temática exerce papel preponderante para a educação populacional. Assim, nesse estudo, objetivou-se compreender a produção científica disponibilizada no Portal da CAPES acerca da educação em saúde no Brasil durante a pandemia da COVID-19. Para tanto, procedeu-se um estudo qualitativo, amparado metodologicamente no estado da arte. De 26 artigos localizados, o corpus do estudo foi constituiu por oito produtos. Analisaram-se os resultados pelo método proposto por Bardin, tornando factível a construção de duas categorias temáticas: desafios impostos à educação em saúde; e estratégias de educação em saúde durante a pandemia da COVID-19. As discussões sinalizaram para o aumento dos desafios na educação em saúde, pelas fake news, em razão da baixa adesão da população às medidas preventivas e devido às vulnerabilidades sociais das quais padecem muitas pessoas. Durante a pandemia, as ações educativas em saúde são fulcrais para manter a capacidade criativa e a sanidade física, emocional e mental. Logo, conclui-se que, o enfrentamento atual exige o envolvimento continuado do Estado, profissionais e sociedade nas estratégias educativas em saúde.
\end{abstract}

Palavras-chave: Pandemia; COVID-19; Educação em saúde.

\begin{abstract}
Health education is an educational process whose scope is to increase the population's autonomy in preventing illness, however the current crisis caused by the COVID-19 pandemic has impacted several social segments. In this context, the dissemination of scientific information on this subject plays a major role in professional and population education. Thus, in this study, the objective was to understand the scientific production available on the CAPES Portal about health education in Brazil during the COVID-19 pandemic. Therefore, a qualitative study was performed, methodologically supported by the state-of-the-art. Out of 26 articles found, eight were selected and used in the corpus of the study. The results were analyzed using the method proposed by Bardin, making the construction of two thematic categories feasible: challenges imposed on health education; and health education strategies during the COVID-19 pandemic. The discussions signaled an increase in the challenges in health education, due to fake news, due to the low adherence of the population to preventive measures, and due to the social vulnerabilities from which
\end{abstract}


many people suffer. During the pandemic, health education actions are essential to maintain people's creative capacity along with physical, emotional, and mental health. Therefore, it is concluded that the current battle requires the continuance of the involvement of the State, professionals, and society in educational strategies in health.

Keywords: Pandemic; COVID-19; Health education.

\section{Resumen}

La educación en salud es un proceso educativo cuyo alcance es incrementar la autonomía de la población en la prevención de enfermedades, sin embargo, la actual crisis impuesta por la pandemia COVID-19 ha impactado a varios segmentos sociales. En este contexto, la difusión de información científica sobre este tema juega un papel importante en la educación en población. Así, en este estudio, el objetivo fue conocer la producción científica disponible en el Portal CAPES sobre educación para la salud en Brasil durante la pandemia COVID-19. Por ello, se realizó un estudio cualitativo, metodológicamente respaldado por el estado del arte. De los 26 artículos encontrados, el corpus del estudio constaba de ocho productos. Los resultados fueron analizados utilizando el método propuesto por Bardin, haciendo factible la construcción de dos categorías temáticas: desafíos impuestos a la educación para la salud; y estrategias de educación para la salud durante la pandemia de COVID-19. Las discusiones señalaron un aumento de los desafíos en educación para la salud, por las fake news, por la baja adherencia de la población a las medidas preventivas y por las vulnerabilidades sociales que sufren muchas personas. Durante la pandemia, las acciones de educación sanitaria son fundamentales para mantener la capacidad creativa y la salud física, emocional y mental. Por tanto, se concluye que el enfrentamiento actual requiere la continua implicación del Estado, los profesionales y la sociedad en las estrategias educativas en salud.

Palabras clave: Pandemia; COVID-19; Educación en salud.

\section{Introdução}

A educação em saúde congrega saberes da saúde e da educação, porque visa instruir a população a respeito da prevenção do adoecimento. Além da construção do conhecimento no âmbito da população, conforme destacam Lima et al. (2020), as ações educativas em saúde executadas pelos profissionais da saúde que estão em formação resultam em benefícios para o profissional, por favorecerem a aquisição de pensamento crítico-reflexivo e a prática fundamentada nas evidências científicas e nas reais necessidades dos destinatários das ações educativas preventivas, de cura e de reabilitação.

Cuidar e educar, concomitantemente, congregam diversos conhecimentos que são construídos e reconstruídos segundo as carências individuais e coletivas das pessoas (Dantas et al., 2020). Mas a pandemia imposta pelo novo coronavírus, Sars-Cov-2 (Acute Respiratory Syndrome Coronavirus 2), assim como em outros campos da sociedade, aumentou quanti e qualitativamente os desafios já existentes na atenção à saúde e aos processos de ensino e aprendizagem.

Se, devido ao aumento exponencial da curva de contágio e do número de doentes graves e fatais, aos hospitais foi requisitado reordenamento do atendimento, aumento da oferta de leitos e acréscimo do aporte de material médico hospitalar, principalmente dos equipamentos de proteção individual (EPI), da população, exigiu-se maior autonomia no cuidado preventivo em saúde; porém, para Palácio e Takenami (2020), diante da rápida expansão das doenças, muitos recebem informações e orientações, e poucos cumprem as recomendações recebidas, podendo, inclusive, haver conflitos e divergências nos saberes e nas práticas de saúde entre a população.

Diante dessa problemática, questionou-se qual é a produção do conhecimento acerca da educação em saúde no atual cenário pandêmico brasileiro? O termo "educação em saúde” foi utilizado conforme a compreensão delineada pelo Ministério da Saúde (MS), a saber:

Processo educativo de construção de conhecimentos em saúde que visa à apropriação temática pela população e não à profissionalização ou à carreira na saúde. Conjunto de práticas do setor que contribui para aumentar a autonomia das pessoas no seu cuidado e no debate com os profissionais e os gestores a fim de alcançar uma atenção à saúde de acordo com suas necessidades (Brasil, 2013, p. 19-20).

Com o objetivo de compreender a produção científica disponibilizada no Portal de Periódicos da Coordenação de Aperfeiçoamento de Pessoal de Nível Superior (CAPES) acerca da educação em saúde no contexto brasileiro durante a 
pandemia da COVID-19, buscaram-se respostas para a questão condutora por meio um estudo qualitativo amparado no estado da arte, uma vez que tal metodologia permite conhecer a produção existente sobre a temática, o que está mais evidenciado, a metodologia utilizada, os objetivos traçados e os principais resultados obtidos. Ademais, permite identificar as eventuais lacunas do conhecimento, incentivar novos estudos e esclarecer o problema investigado (Ferreira, 2002). Optou-se pelos produtos disponibilizados pela CAPES, porque essa biblioteca reúne produtos das pesquisas científicas de todo Brasil.

\section{Metodologia}

O estudo é qualitativo (Minayo, 2012) e embasado no estado da arte (Ferreira, 2002), porquanto, o interesse não foi apenas identificar e mapear a produção preexistente sobre a temática, mas também analisá-la seguindo a técnica de análise de conteúdo (Bardin, 2016). Para Minayo (2012), estudos qualitativos propiciam problematizar teorias e hipóteses, bem como entender, interpretar e refletir valores, opiniões, vivências, condutas humanas e sociais. Já o estado da arte, consoante Ferreira (2002), possui carácter bibliográfico e resulta no mapeamento, descrição e discussão sobre determinada produção científica.

Em julho de 2021, realizou-se busca por assunto, no portal de periódico da CAPES, a partir do acesso disponível para a Comunidade Acadêmica Federada (CAFe) pela Universidade Federal da Paraíba (UFPB), com filtros aplicados da seguinte forma: a) combinações de descritores existentes no DeCs (Descritores em Ciências da Saúde): COVID-19 AND "educação em saúde" e pandemia AND "educação em saúde”; b) localização dos descritores: em qualquer seção dos textos dos artigos; c) restrição de comparação: contém, porque a finalidade era encontrar produtos contendo os citados descritores ; d) data da publicação: últimos dois anos; e) tipo de material: artigos revisados por pares; e f) idioma: qualquer idioma, pois se almejou acessar todos os artigos disponibilizados para a UFPB que contivessem simultaneamente os termos COVID-19 e/ou pandemia e educação em saúde. As aspas foram adicionadas ao termo "educação em saúde" com o propósito de localizar artigos contendo esse termo composto e não apenas "saúde" ou "educação" separadamente.

Foram excluídos os editoriais em geral, artigos repetidos e aqueles contendo o termo "educação em saúde" em sentido diferente ao adotado pelo Ministério da Saúde, isto é, não significativo do processo educativo direcionado à população com a finalidade de promover a autonomia no autocuidado (Brasil, 2013).

Os estudos foram selecionados em duas etapas. Na primeira, das associações dos descritores COVID-19 AND educação em saúde e pandemia AND educação em saúde, excluídos os repetidos, resultaram em 26 artigos, dos quais, após leitura dos títulos, resumos e palavras-chave, 16 seguiram para a fase seguinte. Na segunda etapa, após a leitura detalhada de todo conteúdo dos artigos pré-selecionados, elegeram-se para a constituição do corpus do estudo oito deles, conforme apresentados no item de resultados e discussão.

Aos artigos, foi atribuído um código alfanumérico de "al" a "a8", no qual se aplicou a alínea "a" em referência ao termo "artigo" e os números de 1 a 8 especificaram a ordem do material submetido à análise e a apresentação nos quadros integrantes deste estudo, porque, como orienta Bardin (2016), a análise de conteúdo se organiza em etapas cronológicas: a) pré-análise; b) exploração do material; e c) tratamento dos resultados obtidos mediante inferência e interpretação.

O processo de seleção dos artigos ocorreu por dois revisores independentes e as divergências foram solucionadas por um terceiro revisor.

Optou-se por construir este estado da arte a partir da produção hospedada no portal da CAPES por ser uma vasta biblioteca virtual ligada ao Ministério da Educação (MEC), que reúne produtos científicos nacional e internacional de 130 bases no importe de cerca de 45 mil títulos (Brasil, 2021).

A pesquisa não careceu de aprovação pelo Comitê de Ética em Pesquisa (CEP); mas, em todas as fases do estudo, os pesquisadores respeitaram os princípios éticos e a legalidade, quanto ao rigor científico, autoria e manuseio dos dados. 


\section{Resultados e Discussão}

Da seleção realizada em duas fases, conforme especificado na metodologia, o corpus se constituiu por oito produtos, codificados de "a1" a "a8” segundo título, autoria, ano de publicação, periódico, volume e número especificado no Quadro 1 a seguir.

Quadro 1 - Publicações sobre educação em saúde durante à pandemia da COVID-19 selecionadas no Portal da CAPES 2021.

\begin{tabular}{|c|c|c|c|c|c|}
\hline Cód. & Título & Autoria e ano & Periódico & $\mathbf{V}$ & $\mathbf{N}$ \\
\hline a1 & $\begin{array}{l}\text { Em tempos de pandemia pela COVID-19: o desafio para } \\
\text { a educação em saúde. }\end{array}$ & $\begin{array}{l}\text { Palácio, M. A. V. e } \\
\text { Takenami, I. (2020) }\end{array}$ & Visa em debate & 8 & 2 \\
\hline a2 & $\begin{array}{l}\text { Incertezas em tempos de pandemia: uma reflexão sobre a } \\
\text { educação popular em saúde. }\end{array}$ & $\begin{array}{c}\text { David, H. M. S. L, } \\
\text { Nespoli, G. e Lemos, M. } \\
\text { A. M. (2020). }\end{array}$ & $\begin{array}{l}\text { Revista Educação } \\
\text { Popular }\end{array}$ & Esp & Esp \\
\hline a3 & $\begin{array}{l}\text { Atuação do fisioterapeuta na saúde primária no } \\
\text { enfrentamento da COVID-19: relato de experiência. }\end{array}$ & Figueiredo et al. (2021) & $\begin{array}{l}\text { Revista Brasileira em } \\
\text { Promoção da Saúde }\end{array}$ & 34 & 1 \\
\hline $\mathrm{a} 4$ & $\begin{array}{l}\text { Telepsicologia para famílias durante a pandemia de } \\
\text { COVID-19: uma experiência com telepsicoterapia e } \\
\text { telepsicoeducação. }\end{array}$ & Silva et al. (2020) & Hu Revista & 46 & 1 \\
\hline a5 & The pandemic in prison: interventions and overisolation. & $\begin{array}{l}\text { Carvalho, S. G. de, } \\
\text { Santos, A. B. S. dos e } \\
\text { Santos, I. M. (2020) }\end{array}$ & $\begin{array}{l}\text { Ciência e Saúde } \\
\text { Coletiva }\end{array}$ & 25 & 9 \\
\hline a6 & $\begin{array}{l}\text { Quarentena da COVID-19 no Brasil: necessidade ou } \\
\text { política do excesso contra o desconhecido? Uma crítica } \\
\text { ao plano brasileiro de contingência, frente às orientações } \\
\text { do relatório do Imperial College team. }\end{array}$ & Mamede, W. (2020) & Revista Sustinere & 8 & 1 \\
\hline a7 & $\begin{array}{l}\text { COVID-19 and the impacts on mental health: A sample } \\
\text { from Rio Grande do Sul, Brazil }\end{array}$ & Duarte et al. (2020) & $\begin{array}{c}\text { Ciência e Saúde } \\
\text { Coletiva }\end{array}$ & 25 & 9 \\
\hline $\mathrm{a} 8$ & $\begin{array}{c}\text { Community health workers: Reflections on the health } \\
\text { work process in COVID-19 pandemic times. }\end{array}$ & Maciel et al. (2020) & $\begin{array}{l}\text { Ciência e Saúde } \\
\text { Coletiva }\end{array}$ & 25 & 2 \\
\hline
\end{tabular}

Fonte: Elaboração própria (2021).

Quadro 2 - Detalhamento dos produtos localizados no Portal da CAPES quanto ao tipo de publicação - 2021.

\begin{tabular}{|c|l|}
\hline Cód. & \multicolumn{1}{c|}{ Tipologia } \\
\hline a1 & Ensaio \\
\hline a2 & Artigo de reflexão \\
\hline a3 & Relato de experiência \\
\hline a4 & Relato de experiência \\
\hline a5 & Revisão narrativa \\
\hline a6 & Ensaio \\
\hline a7 & Estudo transversal \\
\hline a8 & Revisão de literatura \\
\hline
\end{tabular}

Fonte: Elaboração própria (2021).

Nos quadros acima, dos artigos qualificados para o estudo, depreende-se que dois são relatos de experiência e compartilham o resultado do trabalho educativo em saúde desenvolvidos por fisioterapeutas (Figueiredo et al., 2021) e psicólogos (Silva et al., 2020), respectivamente; dois classificam-se em ensaios, nos quais os desafios da educação em saúde na pandemia são discutidos (Palácio \& Takenami, 2020) e variáveis como informações científicas, planos, diretrizes, intervenções são debatidas sob a ótica da participação do Estado e do papel da educação em saúde no enfrentamento da pandemia (Mamede, 2020); um artigo de reflexão cuja base teórica se construiu sobre a valorização do saber popular nas práticas de educação em saúde (David, Nespoli \& Lemos, 2020); uma revisão narrativa que preocupou-se com a problemática da COVID-19 nos presídios brasileiros e com as ações governamentais e da sociedade com vistas a reduzir os efeitos adversos 
da pandemia para população carcerárias e agentes de segurança pública (Carvalho, Santos \& Santos, 2020); um estudo transversal quantitativo que debruçou-se acerca dos efeitos do isolamento social sobre a saúde mental e a respeito da importância da educação em saúde na prevenção do contágio pelo Sars-Cov-2 (Duarte et al., 2020); e uma revisão de literatura da qual a educação em saúde desponta como eixo condutor do trabalho dos agentes comunitários de saúde (Maciel et al., 2020).

Considerando que os objetivos delineados nos artigos possibilitam identificar a intensão do investigador ao construir o conhecimento científico, no Quadro 3, especificaram-se os objetivos constantes nos produtos, os quais também embasaram a construção das categorias temáticas analisadas.

Quadro 3 - Especificação dos produtos localizados quanto aos objetivos e à síntese conclusiva.

\begin{tabular}{|c|c|c|}
\hline Cód. & Objetivos & Síntese conclusiva \\
\hline a1 & $\begin{array}{l}\text { Refletir sobre os desafios e as perspectivas de educar em } \\
\text { saúde frente a pandemia da COVID- } 19 \text {. }\end{array}$ & $\begin{array}{l}\text { As medidas de prevenção do contágio pelo novo coronavírus } \\
\text { ainda encontram dificuldade para serem implementadas. }\end{array}$ \\
\hline a2 & $\begin{array}{l}\text { Refletir sobre os sentidos e possibilidades do "popular" nas } \\
\text { práticas de educação em saúde, considerando a atual } \\
\text { conjuntura de incertezas sociais e político-econômicas } \\
\text { agravadas pela incidência da pandemia da COVID-19 no } \\
\text { Brasil. }\end{array}$ & $\begin{array}{l}\text { O momento atual exige uma postura ética-política e } \\
\text { epistemológica produtora de práticas educativas emancipatórias } \\
\text { que valorizem o saber popular. }\end{array}$ \\
\hline a3 & $\begin{array}{l}\text { Relatar a experiência de residentes de fisioterapia em saúde } \\
\text { coletiva no enfrentamento multidisciplinar e } \\
\text { desenvolvimento de ações frente à pandemia da COVID- } \\
19 \text {. }\end{array}$ & $\begin{array}{l}\text { A inserção do fisioterapeuta na equipe contribui para a formação } \\
\text { e a experiência dos residentes fisioterapeutas na educação em } \\
\text { saúde. }\end{array}$ \\
\hline a4 & $\begin{array}{l}\text { Relatar uma experiência em andamento de telepsicologia } \\
\text { para famílias que envolve o uso de telepsicoterapia e } \\
\text { telepsicoeducação. }\end{array}$ & $\begin{array}{l}\text { A telepsicoeducação por cartilhas e podcasts em tempos de } \\
\text { isolamento contribui para a saúde da população. }\end{array}$ \\
\hline a5 & $\begin{array}{l}\text { Revisar e narrar os efeitos da pandemia em presídios e } \\
\text { como governos e sociedade civil têm se organizado a fim } \\
\text { de reduzir as consequências sobre esses locais. }\end{array}$ & $\begin{array}{l}\text { Nas prisões, as ações de educação em saúde devem ser } \\
\text { intensificadas, pois os problemas que exigem medidas sanitárias } \\
\text { mais severas se sobrepõem; mas, por outro lado, medidas como } \\
\text { suspensão de visitas podem agravar a saúde mental da população } \\
\text { carcerária. }\end{array}$ \\
\hline a6 & $\begin{array}{l}\text { Refletir acerca de variáveis aparentemente não valoradas na } \\
\text { luta contra o coronavírus. }\end{array}$ & $\begin{array}{l}\text { O combate a pandemia da COVID-19 exige a melhoria das } \\
\text { informações científicas sobre o contexto brasileiro, } \\
\text { contextualizando com a realidade do país. }\end{array}$ \\
\hline a7 & $\begin{array}{l}\text { Verificar os fatores associados a indicadores de sintomas de } \\
\text { transtornos mental em moradores do Rio Grande do Sul } \\
\text { durante o período inicial da distância social. }\end{array}$ & $\begin{array}{l}\text { A educação em saúde é relevante para a prevenção da infecção } \\
\text { pela COVID-19 e para a saúde mental da população. }\end{array}$ \\
\hline a8 & $\begin{array}{l}\text { Discutir a reorganização processo de trabalho dos agentes } \\
\text { comunitários de saúde como resultado da pandemia da } \\
\text { COVID-19, considerando sua importância como elo entre a } \\
\text { comunidade e os serviços de saúde no campo da atenção } \\
\text { básica. }\end{array}$ & $\begin{array}{l}\text { A COVID-19 requisitou a reorganização do trabalho, processos e } \\
\text { fluxos de assistência à saúde. Nesse contexto, a educação se } \\
\text { apresenta como eixo norteador do trabalho dos agentes } \\
\text { comunitários. }\end{array}$ \\
\hline
\end{tabular}

Fonte: Elaboração própria (2021).

Importa explicar que os desafios e as perspectivas da educar em saúde em cenário inóspito como o atual foram refletidos por Palácio e Takenami (2020);David, Nespoli e Lemos (2020) discutiram a educação popular e a educação em saúde na produção de práticas emancipatórias; Figueiredo et al. (2020) e Silva et al. (2020) relataram experiências educativas e assistências, inclusive com utilização de Tecnologias Digitais da Informação e Comunicação (TDIC); Carvalho, Santos e Santos (2020) revisaram os efeitos da pandemia sobre profissionais da segurança pública lotados nos presídios e sobre população carcerária; Mamede (2020) refletiu sobre as variáveis atinentes ao enfrentamento da COVID-19, dentre elas, a educação em saúde; Duarte et al. (2020) verificaram os indicadores de doença mental e a relevância da educação em saúde durante o isolamento social; e Maciel et al. (2020) discutiram o exercício dos agentes comunitários como educadores em saúde. 
A leitura atenta dos produtos favoreceu a construção de duas categorias temáticas, a saber: 1) desafios impostos à educação em saúde durante a pandemia da COVID-19; e 2) estratégias de educação em saúde durante a pandemia da COVID19, as quais foram discutidas analiticamente através da confluência entre os componentes deste estado da arte.

\section{Categoria 1 - Desafios impostos à educação em saúde durante a pandemia da COVID-19}

A pandemia da COVID-19 projetou múltiplos desafios para os diversos setores sociais, o da saúde e da educação figuram entre os maiores desafios. Especialmente acerca do ato de educar em saúde, Palácio e Takenami (2020, p. 11) aludem que essa importante empreitada, para resultar na autonomia da população no autocuidado, requer reflexões e envolvimento coletivo muito além dos limites das intervenções curativas, porque contempla "a prevenção, proteção, promoção, reabilitação e cuidados paliativos".

Entretanto, conferindo maior complexidade aos problemas influenciadores da educação em saúde, uma avalanche de informações, algumas não confiáveis, passou a fazer parte do cotidiano das pessoas. Decerto, advogam Palácio e Takenami (2020) que as fake news aumentam os desafios para profissionais da saúde e para as autoridades educarem a população sobre as formas de prevenção do contágio pelo novo coronavírus; porquanto, apesar de serem validadas pelos órgãos oficiais de saúde, ainda carecem de adesão por parte da população.

Partindo da premissa de que a educação em saúde se faz com a participação construtiva das pessoas, outra questão apresentada ao debate é a da produção de saber unilateral e vertical, de cima para baixo, com pouca, ou mesmo isenta da participação popular. David, Nespoli e Lemos (2020) refletem sobre o popular na educação em saúde, alertando que a lógica da produção de saberes deve superar o cientificismo. Para além das prescrições de hábitos e comportamentos reputados como saudáveis, as reais condições de vida das populações devem ser consideradas, porque grande parte das pessoas padece historicamente de vulnerabilidades que lhes subtraem o acesso à moradia digna, à educação, à saúde, entre outros.

Outrossim, na dessemelhança do Brasil, determinadas medidas educativas podem ter implementação mais dificultosa em populações específicas. A exemplo da carcerária, onde, para Carvalho, Santos e Santos (2020), a COVID-19 agravou os problemas pré-existentes de desrespeito aos direitos e à saúde dos presos como verificado pela falta de condições sanitárias básicas e pela superlotação.

Nessa categoria, há de se considerar que os reflexos da pandemia, ao atingir as rotinas das pessoas, impactam a saúde mental, aumentam o nível de estresse e o medo, além de causar sofrimento psíquico (Silva et al., 2020). A propósito, vale lembrar que a precarização das condições de trabalho afeta as emoções das pessoas, principalmente daquelas que estão na linha de frente do atual enfrentamento sanitário, como é o caso dos profissionais da saúde, cujo labor exige efetividade nos cuidados aos doentes e, simultaneamente o exercício das atividades educativas em saúde (Neves, Sabino, Assis \& Santos, 2020).

\section{Categoria 2 - Estratégias de educação em saúde durante a pandemia da COVID-19}

A luta contra o novo coronavírus engloba a educação em geral, em particular a educação em saúde, requisitando, perenemente, a prestação positiva do Estado, sobremodo a preventiva. Nesse cenário, campanhas, programas e movimentos sociais devem ultrapassar os limites do tempo e espaço da pandemia e se perpetuar pelo Brasil afora (Mamede, 2020) no tratamento dos problemas populacionais antigos e emergentes em consonância com as diferenças loco-regionais.

Nessa categoria, também foi revelado que os processos de trabalho dos agentes comunitários em saúde, por conhecerem e abordarem as condições sociosanitárias e outras necessidades individuais e coletivas das pessoas, promovem a educação em saúde (Maciel et al., 2020) e, juntamente com os demais profissionais da saúde, ocupam posição decisiva para a obtenção de melhores índices na saúde da população. 
$\mathrm{Na}$ pandemia, as ações de educação em saúde, por serem essenciais para manter a capacidade criativa de produtividade das pessoas, também precisam se voltar para a saúde mental porque, devido às novas regras e hábitos impostos pela alta transmissibilidade da COVID-19, as consequências do isolamento social como perda de renda e emprego, bem como a veiculação midiática de mortos e infectados podem afetar a saúde mental (Duarte et al., 2020). Mais grave ainda, qualquer pessoa com restrição no convívio social está susceptível ao sofrimento psíquico, sintomas psicológicos: estresse, ansiedade, medo, tristeza, podem causar distúrbios mais severos como é o caso de depressão (Pereira et al., 2020), por isso, é importante que o cuidado não se restrinja aos sintomas físicos, ao que está mais visível, mas também se volte para os fatores mais subjetivos.

Além disso, a falta de informações científicas precisas e claras, de certa forma, chancelou o pretexto de errar por excesso, mas as consequências mais discretas, ou de longo prazo, reverberam na saúde mental, principalmente das crianças (Mamede, 2020). Dessa maneira, profissionais atuantes, na qualidade de elo entre comunidade e serviços de saúde, precisam reorganizar o trabalho guiando-se teórica e empiricamente pela educação em saúde valorativa das crenças e da visão de mundo das pessoas, sem se desvincular da conjuntura histórica, cultural e social (Maciel et al., 2020).

Especificamente quanto à pandemia no sistema prisional brasileiro, Carvalho, Santos e Santos (2020) alertam que, nas unidades carcerárias, constatam-se que ações de educação em saúde têm sido negligenciadas, portanto, recomendam intensificação da educação em saúde, inclusive mediante campanhas educativas.

Planejamento a longo prazo, investimento na educação em saúde, fortalecimento do Sistema Único de Saúde (SUS), propagação de informações qualificadas pela veracidade, construção de hábitos favoráveis à saúde, e formação cidadã crítica são preponderantes na recuperação do país pós-pandemia (Mamede, 2020).

Ações educativas com trabalhadores cuja atividade ocupacional pode propagar o vírus, como é o caso da experiência compartilhada por Figueiredo et al. (2021) para reorientar a prática de trabalhadores do setor de limpeza urbana, são medidas que encontram amparo na capacidade humana de aprendizagem e de renovação, não somente no intuito de se adaptar ao novo cenário, mas de superar a problemática e transformar a realidade na qual estão inseridos (Assis et al., 2020). Para mais, a efetividade da educação em saúde exige a ruptura do paradigma cartesiano, determinísticos e prescritivo mediante a compreensão de diversos outros fatores como o político, cultural e ambiental interferem no alcance dos resultados colimados, porquanto as pessoas estão continuadamente em interação com o espaço onde se inserem (Paula et al., 2020).

No mais, por metodologias como a dinâmica de roda de conversa, pode-se abordar o contágio, proteção pessoal e de familiares, uso de equipamentos de proteção individual (EPI) e higiene pessoal (Figueiredo et al., 2021) e o uso da Tecnologias Digitais da Informação e Comunicação, como é o caso das telepsicologia e/ou telepsicoeducação, que promove a autonomia das pessoas para lidar com os conflitos psicológicos durante a pandemia (Silva, 2020), favorecendo a educação em saúde e resultando na capacitação da população fim de reduzir os malefícios acarretados pela pandemia da COVID-19.

Entretanto, na aplicabilidade dessas estratégias, deve considerar que, durante a pandemia, ações educativas para a população podem não alcançar os resultados desejados em razão da exclusão digital limitante do acesso aos recursos midiáticos (Neves, Machado, Fiuza \& Sabino, 2021). Um estudo que se debruçou sobre o processo ensino-aprendizagem por meio remoto durante no primeiro ano de vigência da pandemia também identificou que essa modalidade de ensino é carente de recursos: equipamentos conectados ao provedor de rede de qualidade e habilidade para o manuseio dessas ferramentas (Neves, Valdegil e Sabino (2021). Disso, exsurge o imperativo da implementação de políticas públicas com vistas a promover a equalização, tanto no campo social, como na saúde e no educacional. 


\section{Considerações Finais}

O estudo permitiu compreender a produção científica disponibilizada no portal da CAPES acerca da educação em saúde no contexto brasileiro durante a pandemia da COVID-19, no período 2020 a 2021. Os resultados elucidaram que a pandemia aumentou os desafios para se implementar e manter a educação em saúde, requerendo reflexões e envolvimento de entes públicos e sociedade civil que valorizem o saber popular e sejam emancipatórios.

Dentre os desafios, mencionam-se a baixa qualidade das informações disseminadas pelo país, as notícias inverídicas, as questões emocionais como o medo do contágio e dos resultados mais gravosos pelo adoecimento, a baixa adesão da população às recomendações preventivas procedentes dos órgãos oficias da saúde e o agravamento das vulnerabilidades sociais entre regiões e pessoas, que dificultam o atendimento das medidas preventivas e educativas em saúde.

As estratégias de implementação da educação em saúde mais enfáticas foram a valorização das crenças pessoais e concepção de mundo, compatibilidade das ações com o contexto histórico, social e cultural dos seus destinatários. Ademais, aspiram planejamento, maior investimento, fortalecimento do SUS e das campanhas educativas, inclusive quanto ao equilíbrio de oportunidades no acesso às tecnologias digitais de informação e comunicação.

É relevante destacar que uma das limitações do estudo foi o número reduzido de artigos que atenderam aos critérios de elegibilidade. Nesse quesito, destaca-se a exclusão de produtos pela persistência do conceito de educação em saúde destoante daquele acatado pelo Ministério da Saúde.

Para estudos futuros, recomendam-se novos levantamentos com a ampliação das bases de dados e aprofundamento das reflexões sobre a temática, porque, na pandemia da COVID-19, apesar de sinalizar-se para um certo esmaecimento após o avanço da vacinação, o surgimento de novas cepas do vírus assombra a todos, além disso, a educação em saúde, em sendo promotora da autonomia das pessoas no cuidado, caracteriza-se pelo dever de oportunizar a participação popular e pela necessidade da continuidade das estratégias educativas.

\section{Referências}

Assis, V. D. de Sabino, R. do N, Neves, V. N. S., Santos, S. T. A. dos, Andrade, C. A. de C., \& Rodrigues, J. M. C. (2020). A vocação transformadora da educação popular em saúde. Research, Society and Development, 9(12), e27191211296. https://doi.org/10.33448/rsd-v9i12.11296

Bardin, L. (2016). Análise de conteúdo (L. Antero Neto, Trad.). Edições: 70.

Brasil. Ministério da Saúde. Secretaria-Executiva. Secretaria de Gestão do Trabalho e da Educação na Saúde. (2012). Glossário temático: gestão do trabalho e da educação na saúde Brasília: Ministério da Saúde. http://bvsms.saude.gov.br/bvs/publicacoes/glossario_gestao_trabalho_2ed.pdf.

Brasil. Ministério da Educação. Portal de Periódicos, da Coordenação de Aperfeiçoamento de Pessoal de Nível Superior (CAPES). (2021). Missão e objetivos. https://www-periodicos-capes-gov-br.ezl.periodicos.capes.gov.br/index.php?option=com_pcontent\&view=pcontent\&alias=missao-objetivos\&Itemid=109.

Carvalho, S. G. dos, Santos, A. B. S. dos, \& Santos, I. M. (2020). The pandemic in prison: Interventions and overisolation. Ciencia e Saude Coletiva, 25(9), 3493-3502. https://doi.org/10.1590/1413-81232020259.15682020.

Dantas, D. C., Magalhães, A. G. da C., Ribeiro, Y. C., Diaz, D. P. G., Xavier, B. L. \& Barreto, A. C. M. (2020). Práticas de educação em saúde dos profissionais de enfermagem para o autocuidado de pacientes com colostomia: scoping review. Research, Society and Development, 9(11), e65691110241. https://doi.org/10.33448/rsd-v9i11.10241.

David, H. M. S. L., Nespoli, G. \& Lemões, M. A. M. (2020). Incertezas em tempos de pandemia. Revista de Educação Popular, 259-267. https://doi.org/10.14393/REP-2020-56012.

Duarte, M. de Q., Santo, M. A. da S., Lima, C. P., Giordani, J. P. \& Treniti, C. M. (2020). Covid-19 and the impacts on mental health: A sample from Rio Grande do Sul, Brazil. Ciencia e Saude Coletiva, 25(9), 3401-3411. https://doi.org/10.1590/1413-81232020259.16472020.

Ferreira, N. S. de A. (2002). As pesquisas denominadas “Estado da Arte”. Educ. Soc., 23(79), 257-272. https://www.scielo.br/pdf/es/v23n79/10857.pdf.

Figueiredo, E. A. B., Silva, K. L. S., Luz, H. D. H., Martins, F. L. M., Marçal, M. A. Vitorino, D. F. de M. \& Costa, H. S. (2021). Atuação do Fisioterapeuta na saúde primária no enfrentamento da COVID 19: relato de experiência. Revista Brasileira em Promoção da Saúde, 34, 1-10. https://doi.org/10.5020/18061230.2021.11164.

Lima, D. C., Garcia, M. P., Lima, E. S. de. \& Bezerra, C. C. (2020). Educação em saúde como ferramenta na prevenção de doenças cardiovasculares no Programa de Atenção à Saúde do Idoso. Research, Society and Development, 9(10), e079107382. https://doi.org/10.33448/rsd-v9i10.7382. 
Research, Society and Development, v. 10, n. 12, e311101220360, 2021

(CC BY 4.0) | ISSN 2525-3409 | DOI: http://dx.doi.org/10.33448/rsd-v10i12.20360

Maciel, F. B. M., Santos, H. L. P. C. dos., Carneiro, R. A. da S., Souza, E. A. de., Prado, N. M. de B. L. \& Teixeira, C. F. de S. (2020). Community health workers: Reflections on the health work process in covid-19 pandemic times. Ciencia e Saude Coletiva, 25(2), 4185-4195. https://doi.org/10.1590/1413812320202510.2.28102020.

Mamede, W. (2020). Quarentena da COVID-19 no Brasil: necessidade ou política do excesso contra o desconhecido? Uma crítica ao plano brasileiro de contingência, frente às orientações do relatório do Imperial College Team. Revista Sustinere, 8(1), 274 -299. https://doi.org/10.12957/sustinere.2020.50902.

Minayo, M. C. S. (2012). Análise qualitativa: teoria, passos e fidedignidade. Ciênc. saúde Coletiva, 17 (3), 621-626. https://www.scielo.br/scielo.php?pid=S1413-81232012000300007\&script=sci_abstract\&tlng=pt.

Neves, V. N. S., Sabino, R. do N., Assis, V. D. de, \& Santos, S. T. A. dos. (2020). O impacto da pandemia de COVID-19 nas emoções dos trabalhadores da saúde e na aprendizagem profissional. Research, Society and Development, 9(12), e32391211367. https://doi.org/10.33448/rsd-v9i12.11367.

Neves, V. N. S, Machado, C. J. dos S., Fialho, L. M. F. \& Sabino, R. do N. (2021). Utilização de lives como ferramenta de educação em saúde durante a pandemia pela covid-19. Educação \& Sociedade 42, e240176. https://doi.org/10.1590/ES.240176.

Neves, V. N. S., Valdegil D. de A., \& Sabino, R. do N. (2021). Ensino remoto emergencial durante a pandemia de COVID-19 no Brasil: estado da arte. Práticas Educativas, Memórias E Oralidades - Rev. Pemo, 3(2), e325271. https://doi.org/10.47149/pemo.v3i2.5271.

Palácio, M. A. V., \& Takenami, I. (2020). Em tempos de pandemia pela COVID-19: o desafio para a educação em saúde. Vigilância Sanitária em Debate, 8(2), 10-15. https://doi.org/10.22239/2317-269x.01530.

Paula, S. F. de, Siqueira, H. C. H. de, Medeiros, A. C., Rangel, R. F., Rodrigues, S. T., \& Pedoroso, V. S. M. (2020). Educação em saúde provida pelo enfermeiro ao cuidador à luz do pensamento ecossistêmico. Research, Society and Development, 9(4), e63942854. https://doi.org/10.33448/rsd-v9i4.2854.

Pereira, M. D., Oliveira, L. C. de, Costa, C. F. T., Bezerra, C. M. de O., Pereira, M. D., Santos, C. K. A. dos, \& Dantas, E. H. M. (2020). A pandemia de COVID-19, o isolamento social, consequências na saúde mental e estratégias de enfrentamento: uma revisão integrativa. Research, Society and Development, 9(7), e652974548. https://doi.org/10.33448/rsd-v9i7.4548.

Silva, A. C. do N., Sales, E. M de, Dutra, A. F., Carnot, L. dos R. \& Barbosa, A. J. G. (2020). Telepsicologia para famílias durante a pandemia de COVID-19: uma experiência com telepsicoterapia e telepsicoeducação. HU Revista, 46, 1-7. https://doi.org/10.34019/1982-8047.2020.v46.31143. 